\title{
Nouveaux opérateurs privés du service public de l'emploi
}

Les pratiques des conseillers sont-elles novatrices?

Job seekers guidance within employment and on the way to employment

\section{Sophie Divay}

\section{(2) OpenEdition}

\section{Journals}

Édition électronique

URL : http://journals.openedition.org/travailemploi/3470

DOI : 10.4000/travailemploi.3470

ISSN : 1775-416X

Éditeur

DARES - Ministère du Travail

Édition imprimée

Date de publication : 30 septembre 2009

Pagination : $37-49$

ISSN : 0224-4365

\section{Référence électronique}

Sophie Divay, « Nouveaux opérateurs privés du service public de l'emploi », Travail et Emploi [En ligne], 119 | juillet-septembre 2009, mis en ligne le 30 septembre 2011, consulté le 30 avril 2019. URL : http:// journals.openedition.org/travailemploi/3470 ; DOI : 10.4000/travailemploi.3470 


\title{
Nouveaux opérateurs privés du service public de l'emploi: les pratiques des conseillers sont-elles novatrices?
}

\author{
Sophie Divay $\left(^{*}\right)$
}

La question de l'accompagnement vers l'emploi des chômeurs prend de l'ampleur notamment depuis les années 1990, période à partir de laquelle les politiques de l'emploi en France ont favorisé la mise en place de dispositifs et de mesures de plus en plus axés sur le suivi individualisé et personnalisé des chômeurs. Plus récemment, la suppression du monopole théorique de placement de l'ANPE a entraîné l'arrivée de nouveaux commanditaires et opérateurs privés de placement (OPP) sur le marché de l'accompagnement. Sans chercher à alimenter les débats qui ont émergé sur l'efficacité de ces nouveaux intervenants, cet article rend compte des résultats d'une enquête menée auprès d'OPP portant sur les pratiques des conseillers analysées à l'aune de trois questionnements transversaux: la sélection des chômeurs, les moyens de leur activation et, enfin, la nature des actions menées auprès des offreurs d'emploi.

Depuis «l'invention du chômage» (SALAis et al., 1989), les questions de la catégorisation, du secours ou encore du contrôle des chômeurs se sont toujours posées. La thématique du suivi - ou encadrement de la recherche d'emploi émerge quant à elle au cours des années 1980. Dans le but de favoriser l'insertion ou la réinsertion professionnelle des individus privés d'emploi, l'ANPE diversifie et multiplie son offre de services; des organismes spécialisés voient le jour, comme les missions locales qui ouvrent leurs portes en 1982.

La mise en place de programmes d'accompagnement des demandeurs d'emploi a, par ailleurs, contribué à augmenter le nombre des intervenants en aide à la recherche d'emploi. On citera le Plan d'aide au retour à l'emploi (PARE), l'un des plus conséquents de ces programmes pour lequel l'État a engagé un effort budgétaire d'environ un milliard d'euros par an, à partir de juillet 2001 (CRÉPON, DejemepPe, Gurgand, 2005), auquel il faut ajouter la participation des partenaires sociaux telle que prévue dans la convention Etat-Unédic-ANPE de juin 2001 relative à la mise en œuvre du PARE. L'ANPE n'a pas été en mesure de faire face seule à l'accroissement des services proposés: «Ainsi, fin 2002 , les prestations sous-traitées concernant les demandeurs d'emploi étaient multipliées par 7,6 par rapport à 1998 , et près de 10 si l'on prend en compte les prévisions 2003.» (BALMARY, 2004, p. 94.)

Plus récemment encore, la loi de programmation pour la cohésion sociale de janvier 2005 a accentué la diversification des intervenants en mettant fin au

(*) CAR Céreq Île-de-France, Centre d'économie de la Sorbonne. Université Paris 1 -Cnrs; Sophie.divay@univ-paris1.fr monopole théorique de placement de l'ANPE. Des agences d'intérim, des sociétés de placement, des associations d'insertion ou encore des cabinets de recrutement ont répondu aux appels d'offres des nouveaux commanditaires, émis notamment par l'Unédic ou encore par des conseils généraux, et ont ainsi fait leur entrée sur le marché de l'accompagnement des chômeurs.

Avec l'arrivée de ces «opérateurs privés de placement» (OPP), une deuxième génération de professionnels en aide à la recherche d'emploi est venue s'ajouter à celle des intervenants déjà en activité sur ce marché, intervenants que l'on peut qualifier de «classiques» pour les distinguer des nouveaux arrivants. Une description sera faite plus loin de ces deux générations d'opérateurs, mais on peut d'ores et déjà noter que de nombreux travaux ont été produits, notamment au cours des années 1990, sur les opérateurs classiques (à l'époque souvent désignés comme des intermédiaires de l'emploi). Ces recherches ont entre autres rendu compte des pratiques des conseillers, de leurs interactions avec les usagers ou encore des limites de l'accompagnement des chômeurs en fonction de leurs ressources sur le marché du travail. Les OPP, quant à eux, ont jusqu'à présent surtout fait l'objet d'évaluations quantitatives, centrées sur des indicateurs de retour en emploi, de durée ou récurrence du chômage concernant les chômeurs accompagnés(1).

(1) Voir le «Quatre pages» publié en janvier 2008 conjointement par l'ANPE, l'Unédic et la Dares, intitulé «L'accompagnement renforcé des demandeurs d'emploi. L'évaluation des expérimentations», consultable sur internet à l'adresse suivante: http://www.travail-solidarite.gouv.fr/IMG/ pdf/Evaluation_OPPCVE_n1.pdf. 


\section{Encadré 1}

\section{Caractéristiques des opérateurs privés de placement étudiés}

Dans cet article, sera utilisée une partie du matériel recueilli dans le cadre d'une recherche portant sur «l'accompagnement vers et dans l'emploi » menée en 2007-2008 et commanditée par la Dares. Cette recherche a impliqué la participation de chercheurs de trois équipes: le GREE de l'Université de Nancy 2, le LESSOR de I'Université de Rennes 2 et le Matisse du Centre d'économie de la Sorbonne. Les investigations auprès des OPP présentées ici se sont déroulées sur trois sites d'Île-de-France*:

\section{- OPP Intérim}

Antenne d'un grand groupe spécialisé dans le travail temporaire. L'équipe est composée d'une responsable et de trois conseillers. Au moment de l'enquête, cette équipe venait d'achever un contrat passé avec le conseil général qui visait l'accompagnement de 200 personnes percevant le RMI. Le dispositif a été mis en place début 2006, pour une durée de 12 mois. Les conditions d'accompagnement comprenaient un suivi hebdomadaire par le biais d'entretiens individuels d'une heure. Les trois conseillers, employés à plein-temps, avaient chacun environ 60 chômeurs dans leur portefeuille. Les objectifs de reclassement imposés dans le cahier des charges prévoyaient un taux de $60 \%$ de retour en emploi, en CDI ou CDD d'au moins 6 mois. OPP Intérim est parvenu à reclasser $57 \%$ des chômeurs suivis.

- OPP X1: équipe composée de sept conseillers à plein-temps et d'une manager.

- OPP X2: équipe composée de huit conseillers et demi à plein-temps et d'une manager.

Ces deux structures appartiennent à une société internationale spécialisée dans l'accompagnement et le placement des chômeurs. Au moment de l'enquête, elles sont prestataires de services de l'Unédic.

Le public accompagné comprend des chômeurs percevant l'ARE et ayant des droits ouverts pendant au minimum un an. Ils ont été repérés par leur conseiller ANPE comme des personnes ayant un risque important de devenir chômeurs de longue durée. Ils sont reçus toutes les semaines par leur conseiller référent dans le cadre d'un entretien de $30 \mathrm{mn}$. Chaque conseiller reçoit en moyenne huit personnes par jour et a environ quarante demandeurs d'emploi en portefeuille. Les objectifs imposés aux conseillers sont les suivants: ils doivent effectuer le placement de quatre demandeurs d'emploi par semaine, en CDI ou CDD d'au moins 6 mois; ils doivent également recueillir deux offres d'emploi par semaine.

* Les trois sites enquêtés sont désignés ici sous des noms d'emprunt.

Au-delà des débats focalisés sur les performances quantitatives des nouveaux opérateurs, de nombreuses questions demeurent en suspens: quelles spécificités ou «innovations » en matière d'aide à la recherche d'emploi les OPP peuvent-ils faire valoir? En d'autres termes, quelle plus-value apportent-ils par rapport aux organismes «classiques » appartenant à la première génération de conseillers qui interviennent, pour certains, depuis plus de vingt ans dans le domaine de l'accompagnement? Les «nouveaux opérateurs » ne rencontrentils pas les mêmes difficultés que leurs prédécesseurs? Si oui, parviennent-ils à les résoudre à l'aide de méthodes ou solutions inédites?

Il ne sera pas possible ici de répondre à ces questions pour l'ensemble des OPP. Plus modestement, nous tenterons d'apporter des éléments de réponse qui pourront jeter les bases d'une investigation sur ces nouveaux intervenants qui restera à approfondir. Nous reprendrons pour ce faire différentes recherches évoquées plus haut et réalisées dans les années 1990-2000 auprès de structures classiques d'aide à la recherche d'emploi; certains de leurs résultats feront office d'axes d'analyse des pratiques des OPP que nous avons étudiées (voir encadré 1).

Dans un premier temps, il s'agira d'examiner la question du «tri» des chômeurs qui peut s'opérer d'au moins deux façons: «L'écrémage consiste à refuser de prendre en charge les demandeurs d'emploi les plus difficiles à placer, et le parcage à les accepter en apparence, mais ensuite à ne pas s'en occuper en les conduisant dans une voie de garage. » (GEORGES, 2007, p. 9.) Observés au sein d'agences locales de l'emploi, de missions locales ou encore de PLIE (Houzel, Outin, Ramaux, 2000; Benarrosh, 2000), de tels procédés sélectifs sont appliqués de façon préventive par les conseillers qui écartent ou invisibilisent ainsi des chômeurs pour lesquels ils ne peuvent rien faire, c'est-à-dire auxquels ils ne peuvent rien proposer. Les OPP considérés ont-ils la latitude et/ou besoin de recourir à un tel tri ? Si non, est-ce parce que les conseillers sont contraints d'accepter tous les usagers? Ou parce qu'ils sont incités, par exemple financièrement, à placer les chômeurs les plus en difficulté?

Nous nous intéresserons, ensuite, aux modalités d'accompagnement des OPP enquêtés et, plus particulièrement, à la «force d'activation» des conseillers: ont-ils mis au point des méthodes de mobilisation «innovantes»? Si tel n'est pas le cas, recourent-ils à des technologies sociales utilisées de longue date en aide à la recherche d'emploi et désormais bien identifiées (DivAY, 1999; EBERSOLD, 2001; Frétigné, 2004; Jellab, 1997; Orianne, 2004): engagement moral du chômeur dans l'accompagnement par le biais d'une contractualisation comme la signature d'une charte, instauration d'une 
relation de confiance, techniques de (re)dynamisation et d'individualisation, sur fond d'occultation du contexte économique, travail de normalisation $\mathrm{du}$ comportement, responsabilisation du candidat, voire culpabilisation. Par ailleurs, il sera également intéressant de regarder si les injonctions de «retour rapide en emploi durable», inscrites dans le cahier des charges par les commanditaires, pèsent lourdement sur l'activité des conseillers en OPP qui ont à faire la preuve de leur efficacité sur le marché du placement, en tant que nouveaux entrants.

Enfin, le troisième axe d'analyse des pratiques des OPP sera celui des relations qu'ils entretiennent avec les employeurs. De nombreux auteurs (Belkacem, Charlier, Deshayes, Hirlet, 2001 ; Baron, Bureau, Le Dantec, Nivolle, 1994; Bureau, Marchal, 2005; Castra, 2003; Nivolle, 1999; MEYer, 1998) ayant insisté sur l'insuffisance des contacts entre intermédiaires classiques de l'emploi et entreprises, nous nous pencherons, à leur suite, sur les échanges que les OPP considérés entretiennent avec les employeurs, les éventuels services proposés à ces derniers, ou encore les modes de prospection d'offres d'emploi développés par les conseillers.

Avant d'entrer dans ces développements, sera présenté en préambule l'espace professionnel du champ de l'aide à la recherche d'emploi, description qui permettra de situer structurellement et temporellement les nouveaux opérateurs privés de placement aux côtés des intervenants dits classiques.

\section{Morphologie du champ de l'aide à la recherche d'emploi: trois catégories et deux générations d'intervenants}

Nombreux ont été les observateurs du secteur de l'accompagnement des chômeurs à être pris de vertige face à sa densité et sa complexité. Ce n'est peut-être pas tant la multiplicité des organismes impliqués qui obscurcit le paysage, que l'allure soutenue à laquelle les règles de régulation de ce domaine sont redéfinies. Les modalités de coordination sont sans cesse modifiées par de nouvelles lois et mesures successives qui transforment le rôle des institutions, la nature de leurs interactions ou interdépendances, tout en faisant entrer de nouveaux acteurs dans le jeu. La poursuite d'analyses déjà menées sur la régulation d'un tel système institutionnel (EXERTIER, Gramain, Legal, 2006) pourrait très certainement apporter de riches éclaircissements. On s'en tiendra ici, d'une part, à une identification de la gamme des organismes spécialisés dans le domaine de l'accompagnement visant le placement des individus privés d'emploi et, d'autre part, à un classement en trois catégories de ces orga- nismes permettant de les distinguer, sans pour autant parvenir à un degré de finesse d'analyse qui mettrait en lumière les liens d'interdépendance existant entre les différents segments du champ (relations de cotraitance ou de sous-traitance, partenariats temporaires au travers de conventionnements, etc.) ou encore les situations de chevauchement d'intervention (notamment à travers le «partage» d'un même public cible).

Dans une première grande catégorie, peuvent être regroupées les institutions dont la mission centrale relève soit du placement, soit plus largement de l'insertion professionnelle, notamment par le biais de la formation. On compte, parmi elles, l'ANPE (2) et l'AFPA(3) qui incarnent le Service public de l'emploi (SPE) et qui sont, à ce titre, en charge de la mise en œuvre des politiques de l'emploi. Le tableau s'étoffe avec l'ajout, d'une part, des cotraitants de l'ANPE qui comprennent l'APEC (4), les missions locales et Cap Emploi(5), et, d'autre part, de structures municipales ou des PLIE(6) qui passent des conventions entre autres avec l'ANPE.

Une deuxième catégorie comprend l'ensemble des structures dites «sous-traitantes» qui répondent notamment à des appels d'offres de l'ANPE. D'après un rapport commandé par le Commissariat au Plan (Balmary, 2004), on comptait 4300 prestataires habilités en 2004. Ils se répartissent dans quatre types de statuts: organismes de formation privés, organismes de formation publics ou parapublics, associations des secteurs social et socioculturel, cabinets et associations de conseil (CHEVRIERFATÔME, SimONIN, 2004).

Une troisième et dernière catégorie rassemble des conseillers souvent oubliés ou moins visibles, sans doute parce qu'éloignés ou totalement indépendants du SPE. Ils sont les plus difficiles à repérer du fait de leur dispersion et de leur activité principale qui tend à masquer les services d'accompagnement qu'ils dispensent pourtant. Ainsi en est-il de certaines grandes écoles, universités ou IUT (TRALONGO, 2008) où sont mis en place des modules d'aide à la recherche de stages et d'emploi, voire des cellules pérennes dotées de personnels spécialisés. On citera encore des organismes spécialisés dans l'accompagnement de publics ciblés et financés par des institutions représentantes de ces publics ou d'un secteur d'activité spécifique. Certaines associations dont les services sont destinés aux jeunes diplômés sont ainsi soutenues par des mutuelles étudiantes, d'autres

(2) Il ne sera pas question ici du Pôle Emploi, l'enquête ayant eu lieu courant 2007 et 2008

(3) AFPA: Association nationale pour la formation professionnelle des adultes.

(4) APEC: Association pour l'emploi des cadres.

(5) Cap Emploi: Réseau de 119 organismes spécialisés auprès des personnes handicapées en démarche d'accès ou de retour à l'emploi en milieu ordinaire de travail.

(6) PLIE: Plan local d'insertion par l'économique. 


\section{Encadré 2}

\section{Présentation globale des 14 salariées interviewées au sein des trois OPP enquêtés}

Les quatorze personnes rencontrées sont des femmes. Trois d'entre elles sont des responsables d'équipe (une par OPP).

Elles portent le titre de manager uniquement chez OPP X (1 et 2).

Elles ont entre 25 ans et 45 ans. Toutefois une différence est observable entre les conseillères de ces OPP:

-à OPP Intérim: les trois conseillères ont entre 45 et 35 ans;

-à OPPX (1 et 2), dix conseillères ont entre 25 et 35 ans. Une seule est âgée de 40 ans.

Dernier diplôme obtenu (information manquante pour la responsable d'OPP Intérim):

- dans le commerce, en économie ou gestion: un BTS action commerciale, trois diplômes de troisième cycle d'école de commerce, un master comptabilité-gestion et commerce international, un master en économie-gestion, un maîtrise en IAE, un maîtrise d'AES, un licence d'AES;

-en GRH: un DESS en droit du travail, gestion des ressources humaines;

-en sciences humaines: un master en ingénierie de la formation, un DESS en droit spécialisé dans les politiques publiques, un DESS ingénierie de développement social.

Le niveau de qualification des salariées des OPP est globalement élevé:

- au minimum bac $+2=1$ conseillère

- bac $+3=1$ conseillère

- bac $+4=4$ conseillères +1 manager

- bac $+5=5$ conseillères +1 manager

Toutes les conseillères et managers interviewées avaient eu une expérience professionnelle d'au moins deux ans avant d'être embauchées dans leur poste actuel.

Huit d'entre elles occupaient déjà un emploi dans le domaine de l'accompagnement des chômeurs avant de prendre leur poste actuel.

comme l'AREF-BTP(7), créée par un OPCA(8) du bâtiment, s'adressent aux jeunes chômeurs désireux de se professionnaliser dans ce secteur.

C'est au sein de la deuxième de ces trois catégories que peut être rattachée la deuxième génération de structures spécialisées dans l'accompagnement et le placement des chômeurs. La notion de génération a été retenue ici car elle recouvre l'idée d'une rupture qui sépare les intervenants en fonction d'un effet de moment qui introduit de nouveaux principes idéologiques prônant en l'occurrence la «marketisation » (Perez, Personnaz, 2008) de la sphère de l'aide à la recherche d'emploi. Sans entrer dans le détail, ce courant de marketisation, recouvrant l'intention de confier au secteur privé la délivrance d'une partie des services d'accompagnement et de placement, a été initié dès 1998 par la Commission européenne selon laquelle la «modernisation des services publics de l'emploi» passe par l'abandon du monopole de placement du SPE et l'externalisation des prestations d'accompagnement à travers leur prise en charge par des prestataires privés. En France, ces mesures sont appliquées, comme on l'a déjà indiqué, à la suite de la promulgation de la loi de programmation de la cohésion sociale, en janvier 2005, qui ouvre officiellement le marché du place-

(7) AREF-BTP: Association régionale paritaire pour la formation continue dans le bâtiment et les travaux publics.

(8) OPCA: Organisme paritaire collecteur agréé. ment et de l'accompagnement aux opérateurs du secteur marchand (RousseAu, 2005).

Concrètement, la fin du monopole de placement de l'ANPE produit des changements et des réactions dans le champ de l'accompagnement des chômeurs : l'entrée en scène de nouveaux commanditaires de prestations d'accompagnement des chômeurs, notamment l'Unédic et les collectivités territoriales; l'arrivée sur ce marché de nouveaux opérateurs privés comme des agences d'intérim, des entreprises et associations d'insertion, des cabinets conseil, des agences de placement privées; enfin, la production de nombreux débats ou controverses institutionnels et politiques qui expriment des prises de position favorables ou critiques envers ce que l'on a appelé la «marketisation» du champ de l'accompagnement.

Nous n'entrerons pas ici dans ces débats; notre propos se limitera à la présentation de résultats d'une étude des pratiques développées au sein d'OPP que nous avons étudiés (voir encadrés 1 et 2). Ces pratiques seront analysées à l'aune de trois questions, présentées en introduction, portant tout d'abord sur le «tri» des chômeurs jugés «accompagnables» ou non, puis sur la «force d'activation» des conseillers, et enfin sur leurs relations avec les employeurs. L'examen de ces trois dimensions permettra d'établir une comparaison entre les pratiques des opérateurs classiques (déjà analysées par différents auteurs) et celles des OPP enquêtés dont certains apports spécifiques et origi- 
naux pourront être mis en évidence. Ces constats ne sont en rien généralisables à l'ensemble des OPP auxquels l'Unédic ou les conseils généraux font appel depuis 2005 ; ils constituent l'amorce d'une exploration des pratiques de ces organismes et soulèvent sans doute davantage de questions qu'ils n'apportent de réponses; ils doivent, de ce fait, être considérés comme autant de pistes de recherche à approfondir.

Les investigations ont été réalisées au sein de trois antennes rattachées à deux OPP différentes. Le premier site, baptisé OPP Intérim, appartient à un groupe spécialisé dans le travail temporaire. Il a diversifié ses activités en se positionnant sur le marché du placement des chômeurs dès janvier 2005. OPP X1 et OPP X 2 sont les deux autres sites explorés; ils font partie de la société internationale OPP X, récemment implantée en France et spécialisée dans l'accompagnement et le placement des chômeurs. Quatorze entretiens approfondis, enregistrés et intégralement retranscrits ont été menés auprès de responsables et de conseillères chargées de l'accompagnement des chômeurs de ces trois antennes (voir encadré 2). OPP Intérim, au moment de notre enquête, venait d'achever un programme d'accompagnement d'un public percevant le RMI, programme qui s'était étalé sur an, à partir de décembre 2005 et qui avait été commandité par le conseil général. OPP X 1 et 2, pour leur part, poursuivaient la prise en charge du placement de demandeurs d'emploi dans le cadre de la deuxième vague d'expérimentation d'accompagnement renforcé commanditée par l'Unédic fin 2006(9).

\section{La sélection des chômeurs selon les critères d' "accompagnabilité" et d'“employabilité»"}

Si les évaluations des dispositifs d'accompagnement des chômeurs font du taux de retour en emploi le critère central de leurs analyses, elles n'évacuent pas pour autant d'autres aspects susceptibles d'influer sur les résultats enregistrés. Le «biais de sélectivité des programmes» est très souvent évoqué et vu comme un procédé auquel les conseillers recourent, non pas dans l'intérêt des chômeurs, mais pour augmenter leurs chances d'atteindre les objectifs de reclassement fixés par leur commanditaire. Différentes solutions ont été étudiées. Certains auteurs comme FERRACCI (2007) préconisent l'instauration d'un système d'incitations financières susceptible d'améliorer l'efficacité des opérateurs publics ou privés qui seraient ainsi poussés à limiter l'éviction des chômeurs les moins «employables».

(9) Voir le détail de ces expérimentations dans le «Quatre pages» de l'ANPE, l'Unédic et la Dares, 2008, op. cit.
D'autres ont étudié la mise en place du profilage rationalisé des usagers qui, via la mise en place d'outils statistiques informatisés d'allocation des demandeurs d'emploi, sont censés supprimer l'application de critères informels et subjectifs par les conseillers (GEORGES, 2006).

Il ne s'agira pas dans ce paragraphe d'adopter un point de vue normatif sur les pratiques de sélection des conseillers dont les procédés de tri des chômeurs sont parfois jugés sévèrement, notamment par les financeurs qui craignent un détournement de leurs crédits au profit des chômeurs les moins en difficulté. On essaiera de rendre compte de ces pratiques et de comprendre les logiques des professionnels bien souvent confrontés à des contradictions institutionnelles qu'ils doivent résoudre seuls, loin des décideurs et concepteurs des dispositifs qu'ils ont la charge de mettre en œuvre.

\section{Trois modes de "sélection nécessaire" appliqués par les conseillères d’OPP Intérim}

Le cahier des charges du conseil général engageait OPP Intérim à accompagner 200 «bénéficiaires du $\mathrm{RMI}$ » et à remettre en emploi (CDI ou CDD de plus de 6 mois) $60 \%$ d'entre eux. Comme le souligne la responsable, le recrutement des destinataires de cet accompagnement se déroulait «en terrain connu pour eux», c'est-à-dire au sein des maisons de l'emploi de leur secteur. Les assistantes sociales ont joué un rôle important tout au long du déroulement de cette action. Elles ont, en premier lieu, informé les personnes percevant le RMI qu'elles suivaient de l'existence de cette action et les ont incitées à s'y engager. Elles ont, en outre, été en contact avec les conseillères tout au long de l'accompagnement, en faisant notamment partie des comités de pilotage, instances où l'équipe d'OPP Intérim devait régulièrement rendre des comptes sur l'avancée des suivis et sur l'état des situations de chaque destinataire.

D'après la responsable, les «référents sociaux» ont présenté le dispositif d'accompagnement à 496 personnes ; 377 ont assisté à la réunion de présentation; 340 ont ensuite accepté d'être reçues par une conseillère en entretien individuel organisé aussitôt après la réunion collective; finalement, 230 personnes ont accepté d'être accompagnées en signant la charte élaborée par OPP Intérim; en soustrayant les abandons intervenus en cours d'action, 200 personnes ont été réellement suivies et $57 \%$ d'entre elles ont été considérées comme remises en emploi, selon les critères du commanditaire.

Pour les conseillères et leur responsable, cette expérience a été très intéressante, mais également très intense. Elles ont beaucoup apprécié la richesse de la plupart des entretiens et le temps dont elles ont disposé pour suivre les chômeurs dans le cadre des entretiens hebdomadaires, d'une durée minimale d'une heure. Toutefois, elles insistent sur les diffi- 
cultés rencontrées auprès des personnes dites «hors cible», dont les principales caractéristiques sont d'être «non motivées», «pas prêtes à l'emploi » et de présenter des «problématiques lourdes» correspondant majoritairement à des cas d'alcoolisme ou de troubles psychologiques divers perturbant la situation d'accompagnement. De manière préventive, les conseillères, qui menaient les entretiens de premier contact, ont essayé de réaliser une sélection en amont des chômeurs:

\begin{abstract}
«On avait un petit questionnaire, et puis à partir du questionnaire, on leur présentait l'accompagnement: en quoi ça consiste, l'engagement que c'était pour eux, le fait de faire des recherches. Enfin que c'était un investissement, qu'il fallait être acteur, et donc, bon, on n'essayait pas de leur faire peur, mais donc déjà qu'ils soient bien au fait de ça. Et s'ils étaient d'accord et si on jugeait que la personne... bon, à la limite si on voyait qu'elle avait l'air plutôt alcoolique, ou des choses comme ça, parce qu'on a eu aussi des cas de personnes qui buvaient et qu'on a accompagnées, on essayait quand même de pas les faire rentrer parce que c'était difficile de faire travailler quelqu'un qui était alcoolique! On regardait depuis quand la personne était en RMI, y a des indicateurs aussi, c'est pas parce qu'elle est ancienne, mais, bon, l'idée, c'était quand même d'accompagner les gens qui étaient proches de l'emploi, donc de voir depuis quand la personne était au RMI, est-ce qu'elle a eu des entretiens, est-ce qu'elle fait des recherches?» (OPP Intérim, conseillère)
\end{abstract}

Elles ont également été parfois contraintes de faire sortir du dispositif des personnes avec qui elles ne pouvaient pas instaurer une relation conforme à la situation d'accompagnement:

«-Y a des gens qui sont arrivés pour la première fois, on leur a dit les règles, par exemple: "Vous avez pas le droit de fumer dans les locaux." Bon, il est allé fumer dans les toilettes! (rire) Un type qui avait presque 50 ans! Et puis, il sort et il voit une mobylette dans la cour, il dit: "Je vais peut-être aller la chourer!" On s'est dit, là, il est pas du tout dans les clous! C'est pas la peine. Donc, on a tout de suite arrêté parce qu'on s'est dit qu'il faut aussi d'emblée qu'il y ait des choses qui puissent coller, quoi! Si dans le B A BA, il n'est pas capable de respecter les règles, c'est pas possible!

-Et dans ce cas-là, qu'est-ce que vous avez fait?

- On a appelé en fait la maison de l'emploi et on a prévenu l'assistante sociale. C'est pas nous qui prenons la décision d'arrêter le contrat, mais on leur signale et eux la prennent en général, ils sont de notre avis hein, donc pour des cas comme ça, c'est très objectif, dans ces cas-là, on arrête la prestation. » (OPP Intérim, conseillère)

L'éviction en cours d'accompagnement connaît toutefois des limites. Ce procédé est notamment assez mal perçu par les assistantes sociales qui insistent pour donner une chance à certains des allocataires du RMI, même s'ils sont aux prises avec de grandes difficultés. Elles objectent d'ailleurs aux conseillères que si ces gens n'avaient pas autant de problèmes, ils ne feraient pas partie de leur «public RMIste». Ces travailleuses sociales jouent donc un rôle «contre-sélectif» auquel les conseillères ne peuvent guère s'opposer puisque leurs interlocutrices représentent le conseil général, leur commanditaire. Toutefois, il reste encore la possibilité aux conseillères d'effectuer un filtrage invisible aux yeux des commanditaires, simplement en consacrant moins de temps aux personnes qui les amènent à «tourner en rond » :

«Après, bon, bah, si vous voulez, on est obligé, pas de faire le tri, mais bon, on se dit, bon aller, entre la personne qui est hypermotivée, qui fait tout et qui effectivement a du mal à trouver parce que voilà, c'est le marché qui est comme ça, on va tout faire pour l'aider. Et puis, la personne qu'on a déjà aidée, mais qui s'en fiche, bon, elle, on la voit moins souvent et on offre plus de temps à la personne qui en a besoin et qui veut vraiment bosser! (OPP Intérim, conseillère)

\section{Quand la sélection des chômeurs n'est pas nécessaire}

Selon les conditions du cahier des charges, OPP $\mathrm{X} 1$ et 2 accompagnent des chômeurs inscrits depuis moins de 6 mois à l'ANPE; ces derniers perçoivent l'allocation d'aide au retour à l'emploi et ont des droits ouverts pour au minimum un an. Ils sont envoyés par leur conseiller référent de l'ANPE qui a jugé bon de leur proposer un «accompagnement renforcé», estimant qu'ils présentaient un risque de chômage de longue durée. La réunion dite «de lancement» a lieu dans les locaux du prestataire de service. Les managers des deux sites enquêtés ont fait le constat qu'il leur était souvent difficile de remplir le portefeuille des conseillères, sachant que tous les chômeurs n'acceptaient pas l'accompagnement proposé et qu'il leur fallait de ce fait se montrer persuasifs pour retenir le maximum de personnes.

Aucune des onze conseillères et responsables d'OPP X1 et 2 interviewées n'a fait état de procédés de sélection des chercheurs d'emploi. Elles ont au contraire détaillé les arguments qu'elles déploient pour convaincre les chercheurs d'emploi d'entrer dans le dispositif:

\begin{abstract}
"L'objectif, c'est que les personnes adhèrent au programme parce qu'on est là pour les aider à retrouver un emploi, mais à partir du moment où les personnes sont volontaires et ont envie de trouver un emploi, y a pas de raison! Et c'est ce qu'on leur dit: "Le seul risque que vous avez, c'est de retrouver un poste. La contrainte des rendez-vous toutes les semaines, c'est pas une contrainte, c'est: plus on va se voir, plus y a de chances que vous soyez en poste!" On va pas forcer les personnes, on va leur faire comprendre leur intérêt de bénéficier de notre accompagnement. » (OPP X1, manager)
\end{abstract}

Elle sait par ailleurs quelle réponse faire aux chômeurs récalcitrants : 
«Aux personnes qui refusent l'accompagnement, on leur dit aussi qu'elles vont devoir s'expliquer à l'Assedic: "Bah, pourquoi vous avez refusé?" Et les personnes s'expliquent. Après les conséquences, c'est l'Assedic qui gère, qui décide... Nous, on fait juste un transfert d'information sur si la personne a accepté ou refusé. » (OPPX1, manager)

Le déploiement de l'ensemble de ces tactiques ne signifie pas que les conseillères ne rencontrent aucune difficulté avec les demandeurs d'emploi. Elles tracent d'ailleurs le même portrait du «mauvais client»: il n'est pas motivé; il souffre d'addiction, souvent à l'alcool, ce qui le rend peu présentable à un employeur, notamment parce qu'il porte sur lui les stigmates de sa déviance, par exemple, en sentant l'alcool avant de se rendre à un entretien. Ces aspects comptent parmi les plus gênants pour le conseiller qui sait, d'une part, qu'ils font partie des sujets personnels délicats à aborder avec la personne, et, d'autre part, parce qu'il n'a aucun moyen de les corriger ou de les masquer. Par définition, son intervention est par avance vouée à l'échec. Les conseillères d'OPP X1 et 2 soulignent toutefois que ces cas sont minoritaires dans l'ensemble de leur public et que, de ce fait, ils ne leur causent que peu de soucis.

OPP Intérim et OPP X1 et 2 donnent donc à voir deux cas de figure contrastés. Les conseillères du premier organisme disposent d'une certaine marge de manœuvre pour procéder à un «tri nécessaire des chômeurs », tri nécessaire dans le sens où sans ce recours, elles ne parviendraient pas à atteindre les objectifs de reclassement imposés dans le laps de temps imparti. Les conseillères soulignent d'ailleurs qu'elles ont tout de même eu à traiter une part non négligeable de cas difficiles pour lesquels elles n'ont pas été d'une grande utilité:

«On avait aussi $30 \%$ de gens à accompagner qui n'étaient pas du tout dans la cible, qu'on a quand même intégrés, il a fallu quand même aussi faire pas mal de psy, de choses qui n'étaient pas notre travail, euh... mais bon, ça en a aidé certains. On est content d'avoir pu le faire ça, même si c'était pas notre travail de base, euh... si on peut en sauver un, c'est toujours ça. Mais, non, après, fallait quand même qu'on reste, nous, sur l'emploi, parce que si on commençait à partir dans... ça aurait pu être bien, mais à ce moment-là, il aurait fallu avoir un accompagnement bien plus long!»(OPP Intérim, conseillère)

Les conseillères du second organisme ont, quant à elles, une faible marge de manœuvre pour opérer une sélection des chômeurs, mais leurs conditions d'accompagnement ne nécessitent pas un tri des chômeurs pour lesquels un soutien de la recherche d'emploi suffit souvent à faciliter leur remise en emploi.

\section{Les modalités de l'activation: sur les chômeurs, sur les conseillers}

D'après VRancken et Macquet (2006), un nouveau mode de gouvernement des personnes se répand dans la société, non seulement auprès des plus vulnérables, mais également dans l'ensemble de la population. Une mutation de la question sociale s'est produite notamment en réponse au chômage de masse et à la montée du néolibéralisme. Selon ces auteurs, l'État ne s'est pas retiré, mais a mis en place de nouveaux dispositifs publics qui reposent sur l'activation des personnes pour, entre autres, colmater les brèches du système assurantiel. L'objectif est de renforcer la «capabilité des individus» ou leur autonomie grâce à des dispositifs d'accompagnement qui privilégient une intervention sur autrui, un travail sur soi psychologisant fondé sur la parole, le récit d'une histoire individuelle, favorisant la construction d'un projet. Dans le domaine de l'insertion professionnelle, les conseillers incitent les chômeurs à devenir des «entrepreneurs» de leur carrière et de leur employabilité (EBERSOLD, 2001).

Comme on va le voir, les opérateurs privés de placement sont, au même titre que les conseillers de la première génération du champ de l'accompagnement, confrontés à des difficultés inhérentes à la mobilisation des subjectivités, mais ils sont amenés à y faire face différemment en fonction de leur cadre organisationnel.

\section{OPP Intérim: \\ une activation fondée sur l'écoute}

Les conseillers d'OPP Intérim avaient en moyenne soixante chômeurs dans leur portefeuille, qu'ils rencontraient en entretien individuel d'une heure, toutes les semaines. Cet accompagnement vers l'emploi s'étalant sur douze mois, une certaine proximité s'est progressivement installée entre la plupart des chômeurs et les conseillères, et ce d'autant que les premières étapes de l'aide à la recherche d'emploi démarrent classiquement par la réalisation d'un bilan, c'est-à-dire un récit du parcours professionnel et personnel qui servira de base à l'élaboration d'un projet «réaliste et réalisable», selon une formulation bien connue des conseillers (10). Cette offre d'écoute et de disponibilité a souvent été appréciée par les chômeurs qui après une période d'isolement ont pu trouver une oreille attentive et déverser une part de leurs souffrances, plaintes, récriminations et parfois agressivité. Les conseillères ont de ce fait été émotionnellement mises à l'épreuve : 


\begin{abstract}
«Donc, c'est vrai qu'on sort un peu du créneau, alors c'est sûr qui faut pas non plus, on fait pas du social, on n'est pas assistante sociale, mais c'est vrai qu'on a été confronté quand même à des cas euh... quand même assez difficiles, et c'est vrai que la surprise, c'est qu'on s'y attend pas au départ! (rire) [...] Bah oui, c'est au début que ça a été le plus difficile, quoi! Parce que c'est vrai selon les personnes, y en a qui nous déchargeaient tout, et puis qui étaient vraiment dans des situations difficiles! Déjà, comme ce sont des personnes qui souvent ne parlent pas beaucoup, elles se déchargent sur une personne qui les écoute, après chacun raconte son histoire à sa façon, donc c'est vrai qu'il faut savoir en prendre et en laisser. Et puis, on est touché aussi... parce que la façon dont peuvent s'exprimer les personnes aussi... Donc, il faut être à la fois humain, mais en même temps, faut pas être trop... faut pas avoir un effet miroir, parce que ça, c'est pas bon!»(OPP Intérim, conseillère)
\end{abstract}

Tous les facteurs sont ici réunis pour que la situation d'accompagnement s'apparente à un suivi psychothérapeutique (11): individualisation de l'accompagnement, durée d'entretien d'une heure, invitation à parler de soi, accompagnement programmé sur une année avec un même «accompagnateur» référent. Une telle psychologisation de la relation a parfois amené les conseillères à devoir « redresser la barre», c'est-à-dire à enjoindre à l'usager de passer à l'action, sans hésiter parfois à prendre en charge pour eux une part du travail de recherche d'emploi en appelant directement les employeurs ou en rédigeant le $\mathrm{CV}$ et la lettre de motivation.

\section{OPP X1 et 2: une activation fondée sur "le faire"}

Les conseillères d'OPPX 1 et 2 sont tenues de suivre un flux d'une quarantaine de demandeurs d'emploi et de les recevoir toutes les semaines dans le cadre d'entretiens de 30 minutes. Elles sont censées appliquer un protocole qui standardise les pratiques des conseillères:

«- En entretien individuel, nos limites, c'est: un entretien doit durer une demi-heure et on doit avoir fait le point, alors proposer un café, fait le point sur les objectifs de la semaine précédente! (rire)

- C'est aussi précis que ça?

-Ah, oui, oui: proposer un café, faire le point sur les objectifs de la semaine précédente, ensuite proposer des choses, éventuellement prendre son téléphone pour démarcher, et refixer des objectifs pour la semaine d'après, fixer le rendez-vous et raccompagner jusqu'à la porte. Le protocole, c'est ça! »(OPP X1, conseillère)

Dans cette société, les pratiques des conseillères sont définies à l'aide de nombreux «standards» d'activité et de principes dont l'un des plus fonda-

(11) Voir parmi bien d'autres OrianNe (2004) ou Coutant (2005). mentaux insiste sur le fait que l'accompagnement ne relève pas du travail social. Les clients ou chercheurs d'emploi doivent être traités avec le plus grand respect, mais en aucun cas considérés comme des «assistés». De ce fait, les entretiens doivent être centrés sur la recherche d'emploi, les actions à mener, la prospection, et non pas sur le vécu et les problèmes personnels des individus :

«On nous demande, par exemple, sur un rendezvous d'une demi-heure, de prendre juste le temps de traverser la salle pour parler avec la personne, après c'est pour la recherche d'emploi! » (OPP XI, conseillère)

En prenant connaissance de ces consignes, on comprend pourquoi la formation délivrée aux nouveaux conseillers ne contient pas d'apport sur la conduite d'entretien. Il leur est avant tout proposé une présentation des nombreux outils de travail ou «produits uniques, rationalisés et normalisés » qu'ils auront à utiliser : procédure du premier accueil, déroulé de la réunion de lancement, fiche récapitulative d'accueil de nouveaux candidats, plan d'action des six premières semaines enclenchant la dynamique de recherche, dossier informatisé du candidat, formulaires de reporting hebdomadaires et mensuels, etc.

Les conseillères interviewées soulignent le malaise que génèrent la formalisation de ces prescriptions et les contradictions de cette conception «dépsychologisante» de l'entretien (DivaY, 2008); alors qu'elles travaillent «sur de l'humain », auprès de personnes au chômage, elles doivent limiter le contenu des échanges aux questions techniques et concrètes de la recherche d'emploi, cadre dont les «clients» débordent systématiquement. Il leur incombe alors de faire face seules aux difficultés auxquelles elles se trouvent inévitablement confrontées:

«Hormis les techniques de recherche d'emploi, moi, personnellement, j'avais jamais conduit d'entretien de ma vie avant, quoi! Donc, le premier candidat, on gère! Et puis, on est plus ou moins aidé par le manager... Mais l'avantage, c'est que du coup, on est peut-être un peu plus créatif aussi? En fait, c'est contradictoire, c'est que les conditions de travail sont superrigides, enfin moi je trouve, y a un cadre très fort, y a une pression très forte, mais par contre sur le poste, c'est: "Démerde-toi!"》(OPP X1, conseillère)

OPP Intérim et OPP X doivent donc en grande partie leur «force d'activation» à une intensification de l'accompagnement des chômeurs; en effet, par rapport aux organismes classiques d'aide à la recherche d'emploi, ils proposent un suivi plus fréquent et plus soutenu des usagers: entretiens hebdomadaires entre lesquels s'ajoutent des appels téléphoniques ou messages électroniques. En ce qui concerne les modalités relationnelles d'accompagnement, les conseillères d'OPP Intérim font face à des difficultés semblables à celles que leurs 
collègues rencontrent dans des PLIE ou structures sociales dédiées aux chômeurs en grande difficulté. Ces difficultés les amènent à faire les frais des conséquences humainement éprouvantes de l'individualisation et de la psychologisation de l'accompagnement (CASTRA, 2003), notamment du fait des efforts de redynamisation qu'elles doivent développer pour soutenir moralement leurs interlocuteurs.

À OPP X1 et 2, les modalités d'activation relationnelles pèsent davantage sur les conseillères que sur les demandeurs d'emploi. Soumises à une rationalisation de leur travail prescrit, elles sont obligées d'amortir la rigidité des principes organisationnels dépsychologisants qu'elles ne peuvent appliquer en situation de travail réelle, alors qu'elles sont face à des chômeurs qu'elles ne peuvent empêcher d'exprimer leurs émotions et problèmes personnels.

\section{Travail auprès des offreurs d'emploi dans le cadre de l'accompagnement des demandeurs d'emploi}

Les professionnels du placement et de l'accompagnement des chômeurs ont souvent été désignés sous le nom d' «intermédiaires de l'emploi». Ce qualificatif souligne la dualité de leur intervention, théoriquement orientée tout à la fois vers les offreurs et les demandeurs d'emploi. Des auteurs ont remis en question cette double orientation et démontré le caractère unilatéral des actions développées dans le cadre de la politique publique de lutte contre le chômage: «Désormais, tout est fait pour réduire les dépenses passives et les remplacer par des mesures actives, de plus en plus individualisées, incitatives (voire parfois coercitives) et surtout, tournées quasi exclusivement vers l'offre de travail. Cette logique adaptative repose sur une conception "individualisante" et naturaliste de l'employabilité des chômeurs, qu'il s'agit d'améliorer par tous les moyens pour envisager que ces individus puissent répondre efficacement à une offre d'emploi et passer avec succès un entretien d'embauche. Cette logique met en avant les rôles joués par les travailleurs et les pouvoirs publics. Tout se passe comme si les exigences des firmes étaient des données auxquelles il convient de s'adapter (GAZIER, 2001).» (SALOGNON, 2007).

L'arrivée des agences privées sur le marché du travail ayant été présentée comme une «remise en ordre juridique [...] de nature à favoriser le déploiement de nouvelles stratégies et initiatives » (MARIMBERT, 2004, p. 122), on peut notamment chercher à mesurer leur capacité d'innovation en matière d'action et d'intervention auprès des employeurs.

\section{Une intermédiation déplacée}

OPP Intérim dispose d'une action spécifique dont la responsable du site souligne l'originalité et l'efficacité :

"En fait, ce qui nous différencie d'autres cabinets, c'est d'avoir cette capacité à utiliser le travail temporaire pour tester. On ne part pas seulement sur du CDI ou du CDD, mais on a aussi le temps d'utiliser l'intérim, et puis pour certains ça commence par une mission et il peut y avoir des suites derrière. » (OPP Intérim, responsable)

Le recours au travail temporaire est utilisé dès que possible par les conseillères qui cherchent ainsi à redonner espoir aux chômeurs dès le début de l'accompagnement (12). Cette forme de redynamisation vise à renforcer leur adhésion au dispositif et leur engagement dans la recherche d'emploi. Pour ce faire, elles jouent un rôle d'intermédiaire, non pas entre les entreprises et les demandeurs d'emploi, mais entre ces derniers et les agences intérimaires du réseau régional d'OPP Intérim. Ces démarches nécessitent certes une bonne connaissance des agences du réseau et notamment de leur spécialisation dans certains secteurs d'activité, mais avant tout de l'opiniâtreté et de la diplomatie pour contrer les résistances des collègues d'agence qui tentent de satisfaire les exigences de leurs clients. Conseillères en accompagnement et consultantes en agence, bien que faisant partie de la même société, défendaient souvent des intérêts divergents :

"Y a des agences qui ont joué le jeu, d'autres non. Pour certains profils, c'était pas la peine! Donc, elles nous disaient: "Écoute, moi j'ai un client qui veut que la nana elle ait un bac +4 , trois ans d'expérience, qu'elle maîtrise tel et tel logiciel. Oui, ta candidate, OK, elle a un bac +4 , mais elle ne maîtrise pas les logiciels et puis elle a pas trois mois, mais un an de chômage! Moi, mon client, il en veut pas!" Donc, bah, face à ça, on pouvait pas non plus faire le forcing, hein! Certaines agences ne recevaient même pas nos candidats parce que d'emblée, elles nous disaient: "Écoute, moi rien qu'avec le $C V$, là, je vois qu'il a pas travaillé depuis 2000, mes clients, c'est pas la peine! Déjà, quand y a six mois de non-travail, ils me prennent la tête donc euh... six ou sept ans, ça va pas le faire!" » (OPP Intérim, conseillère)

Au-delà de leur volonté de redonner confiance aux chômeurs, en leur démontrant qu'ils étaient «encore» employables, malgré parfois une longue période de chômage, les conseillères ont également testé leur mobilisation à travers l'expérience en intérim, interprétant les «échecs» comme le signe de dysfonctionnements personnels. Cette instrumentalisation du passage en intérim repose notam-

(12) D'après la responsable du site, $53 \%$ des chômeurs ont eu accès à au moins un contrat intérimaire pendant leur accompagnement. Il n'a pas été possible d'avoir d'informations sur les caractéristiques de ces chômeurs. 
ment sur l'accentuation de l'individualisation et de la responsabilisation du chômeur:

«Y a des gens qui sont passés par le travail temporaire. On l'a utilisé et pour certains c'était une façon très positive de montrer que tout allait bien et qu'on pouvait aller vers quelque chose de plus pérenne, en termes de recherche. Et puis, pour d'autres, ça révélait des difficultés. Donc là, charge au consultant d'analyser, de debriefer avec le bénéficiaire pourquoi ça avait pas fonctionné, pourquoi y avait eu rupture, pourquoi il n'y était pas allé aussi dans certains cas, et travailler sur ce côté-là, sur le versant motivation, et puis comment dépasser aussi ces difficultés-là.» (OPP Intérim, responsable)

En dehors des échanges instaurés avec les agences intérimaires de leur réseau, les conseillères ont établi des contacts directs et ponctuels avec des employeurs en prospectant le marché du travail. Elles se substituaient en quelque sorte aux chômeurs pour accélérer ou redoubler leurs recherches d'emploi. Leur société ne leur ayant fourni aucune liste d'employeurs, elles n'ont pu que constituer progressivement un carnet d'adresses personnel où elles consignaient les noms d'interlocuteurs clés de quelques entreprises:

\begin{abstract}
"On fait aussi notre propre prospection à nous, c'està-dire que, nous, on recherche des offres, on y répond, on essaye quand y a juste le nom de la société de regarder dans les pages jaunes pour trouver le téléphone, on appelle... Enfin, y a tout un travail de prospection, on comptait pas que sur les agences! » (OPP Intérim, conseillère)
\end{abstract}

\section{Une prospection empêchée}

En ce qui concerne leur intervention auprès des employeurs, les conseillères d'OPPX 1 et 2 sont soumises à des objectifs précis qui prévoient le recueil de deux offres d'emploi par semaine. Les exigences sur la nature de ces offres s'avèrent assez souples :

«En fait, vu que nous on est facturable une fois qu'on a mis nos candidats en CDI ou en CDD supérieur à 6 mois, tant qu'à faire, autant trouver des offres de cette durée-là. Seulement, on néglige pas pour autant les CDD inférieurs à 6 mois ou les contrats d'intérim puisqu'ils peuvent permettre un retour à l'emploi, donc les offres, c'est tout type d'offres, en fait.» (OPPX1, conseillère)

Deux heures hebdomadaires sont formellement réservées à la prospection qui s'effectue de deux manières. La plus fréquente relève du principe «faire d'une pierre deux coups »: pendant que les conseillères recherchent des offres pour leurs candidats (en leur présence ou non), elles essaient d'en extraire quelques-unes qu'elles font circuler dans le réseau national de leur société, via leur site intranet. L'autre démarche consiste à entrer en contact avec des employeurs, sans chercher à placer des candidats. Cette méthode est jugée coûteuse en temps et peu rentable. Elle suppose le développement d'un argumentaire commercial(13), l'instauration d'une relation de confiance avec l'interlocuteur, des relances, etc. De surcroît, si l'entreprise signale un poste vacant, OPP X s'engage à donner une réponse (négative ou positive) en 48 heures. La conseillère risque alors d'alourdir sa charge de travail en recherchant un candidat adéquat dans son portefeuille ou auprès de ses collègues. La plupart des conseillères reconnaissent qu'elles ne disposent pas du temps nécessaire pour mener à bien de telles investigations. Par manque de disponibilité, il leur arrive ainsi de rater l'appel d'une entreprise parce qu'elles sont en entretien avec un demandeur d'emploi ; par ailleurs, elles ne trouvent pas les plages de temps nécessaires pour procéder à l'envoi de plaquettes publicitaires après un premier contact téléphonique. En fait, la plus grande partie de leur activité est consacrée à la réception de leurs chercheurs d'emploi, et ce tant par obligation que par goût, beaucoup reconnaissant ne pas apprécier le volet prospection de leur travail :

«-Non, non parce que... je fais pas de prospection! (rire) Voilà, y a des objectifs que j'atteins pas, mais euh... Après les prospections, je le fais plus avec mes candidats en direct. C'est plus simple. Je préfère prendre moins de temps pour la prospection et prendre vraiment le temps de travailler sur le CV, la lettre de motivation, et la prospection de toute façon, on va la faire. Donc, après qu'il le fasse lui, tant mieux, que je le fasse pour lui, je trouve une offre, bah, je le mettrai dans le reporting, mais moi... (baisse la voix) je devrais pas le dire, mais pour moi, c'est pas ma priorité la prospection. Pour certains postes, effectivement, je le fais, parce que boulanger ou agent de sécurité, c'est mieux de faire de la prospection, c'est plus efficace, mais je le fais pas automatiquement.

- Tout dépend des postes un peu?

-Oui, je fais une pierre, deux coups, et je fais la prospection pour les candidats et en même temps la prospection pour atteindre mes objectifs, deux offres par semaine.» (OPPX1, conseillère)

Le «portefeuille entreprises » est de ce fait bien maigre et ne comprend que quelques contacts, et ce même chez les conseillères ayant plus d'un an d'ancienneté dans leur poste:

«-En ce qui vous concerne, vous avez combien d'en-
treprises dans votre portefeuille?

Oh, là, j'en sais rien... parce que moi la prospection, c'est pas un truc dont je raffole! Je suis pas capable de vous dire... (silence) J'ai pas compté... j’ai pas compté...

- Je sais pas, c'est de l'ordre de 10, de 100 ?

(13) Les conseillères mettent en valeur le fait que, contrairement aux agents de l'ANPE, elles ne suivent qu'un nombre limité de candidats, qu'elles connaissent bien. Elles assurent, de ce fait, la délivrance d'un service gratuit et personnalisé aux entreprises auprès desquelles elles ne s'engagent à adresser qu'un nombre limité de candidats adaptés à leurs besoins et dont elles se portent garantes. 
Ah, non, non, c'est une vingtaine de sociétés, oui, oui! »(OPP X2, conseillère)

$\mathrm{Au}$ manque de temps lié à l'organisation et d'intérêt personnel pour cette activité de prospection d'offres d'emploi vient s'ajouter une absence de reconnaissance institutionnelle qui achève de décourager les conseillères. Elles savent qu'elles seront jugées en premier lieu sur leur taux de reclassement des demandeurs d'emploi, résultat dont dépendra directement leur augmentation annuelle de salaire (14):

«On a eu une revalorisation des salaires, c'était quand? Février! Enfin, c'était voilà... et là, c'était en fonction de nos résultats, donc j'imagine... individualisés, mais en fonction des résultats, en fonction de la facturation en tant que notion du taux de placement. Et notre souci, c'est qu'on est sur des activités où, par exemple, quand on prospecte, on prospecte pour tout le monde, donc du coup, on peut perdre du temps, entre guillemets, à prospecter auprès des entreprises, décrocher des offres pour ne pas placer son propre candidat, et ça, ça va pas être valorisé par exemple. La seule chose qui sera valorisée, c'est le placement. » (OPP X1, conseillère)

OPP Intérim et OPP $X$ présentent donc deux modes différenciés d'action auprès des employeurs qui demeurent somme toute bien secondaires par rapport à leur travail consacré aux demandeurs d'emploi. Cet état de fait n'est que la résultante d'une même conception de l'accompagnement partagée par les différents commanditaires: ils font porter l'évaluation des performances de leurs prestataires de service principalement sur les taux de retour en emploi qui impliquent les conseillers et les demandeurs d'emploi, tout en ignorant complètement le comportement des offreurs d'emploi.

$*$
$* \quad *$

À l'issue de l'analyse des pratiques des OPP enquêtés, guidée par trois axes transversaux de questionnement, on retiendra globalement que ces structures ne semblent pas avoir mis au point des méthodes fondamentalement «innovantes», jamais rencontrées ailleurs au sein d'organismes classiques, susceptibles de métamorphoser le champ

(14) Les résultats obtenus par les conseillers jouent donc d'une certaine manière sur la rémunération des conseillers. Mais d'après leurs témoignages, le supérieur hiérarchique qui les évalue tient compte d'un critère objectif, leur taux de reclassement des demandeurs d'emploi, mais aussi d'un critère subjectif, leur comportement (esprit d'équipe, assiduité, ponctualité...). Le nombre d'offres d'emploi recueilli n'intervient donc pas dans leur évaluation. Par ailleurs, leur salaire ne peut varier qu'à la hausse. Aucune conseillère n'a fait état d'une baisse de salaire due à de mauvais résultats. Enfin, il n'a jamais été évoqué l'existence ou le projet d'instaurer une part variable du salaire en fonction des résultats. de l'accompagnement. On observe plutôt des variations dans l'intensité de la mise en œuvre d'opérations ou de stratégies déjà connues.

Les chômeurs jugés non accompagnables et/ ou faiblement «employables» font l'objet d'une sélection par les conseillers des OPP considérés qui recourent à ces stratégies de manière à ce que leur mission d'accompagnement soit «réaliste et réalisable». Ils procèdent à une estimation des paramètres combinés qui définissent leurs chances d'atteindre les objectifs définis par leur commanditaire: durée de la mesure, état du marché du travail local, caractéristiques des chômeurs, fréquence du suivi. Selon la marge de manœuvre dont ils disposent et la recrutabilité (15) de leur public, ils opèrent, lorsque nécessaire, un tri en amont (écrémage) ou en cours de suivi (parcage ou éviction), non pas dans le but de maximiser leurs éventuels profits, puisqu'ils ne sont pas rémunérés selon leurs résultats, mais de manière à remplir leurs obligations quantitatives (taux de placement minimum) et qualitatives, conformes à leur conception d'un accompagnement professionnel et bénéfique pour les chômeurs. Qu'adviendrait-il de ces exigences de qualité si, comme le préconisent certains auteurs (FERRACCI, 2007), étaient instaurées des «incitations financières » récompensant les conseillers les plus performants en matière de taux de reclassement? Tous les chômeurs seraient-ils reclassés? On peut raisonnablement répondre par la négative à cette question. Il est parfois craint que les chômeurs ainsi reclassés soient poussés vers des emplois de mauvaise qualité, en oubliant ceux qui ne seront pas remis en emploi. Or, ceux-là risquent également de voir leurs conditions d'accompagnement se dégrader. Les conseillères ont, en effet, insisté sur les formes d'aide, ne faisant pas partie de leurs attributions(16), qu'elles essaient d'apporter aux chômeurs qu'elles ne parviennent pas, selon leur expression, «à recaser». La mise en place d'incitations financières risquerait alors de pénaliser «les chômeurs non rentables ». Ils pourraient alors ne plus faire l'objet de l'attention dont ils bénéficient aujourd'hui :

"Quand ils sont jeunes, je les laisse jamais, jamais sortir sans solution, je les oriente toujours... même s'ils sont plus âgés, j'oriente sur des choses, et mon petit plus par rapport à mon parcours, au niveau de mes candidats, c'est que quand ils sortent, je sais qu'ils sont autonomes, je mets un point d'honneur au niveau des

(15) Comme le souligne l'auteure de ce néologisme, il «est l'exact synonyme d'employabilité, mais il a l'avantage de renverser la perspective, mettant davantage l'accent sur les pratiques de sélection/exclusion des employeurs envers certaines catégories sociales et non sur les pratiques individuelles de recherche d'emploi» (Pochic, 2007, p. 33).

(16) L'orientation vers la formation ne fait pas partie des objectifs des mesures d'accompagnement étudiées chez OPP Intérim et OPP X qui sont centrées sur le «retour rapide en emploi durable». 
$C V$, au niveau des lettres, parce que j'ai une compétence particulière, et je le sais, là-dessus. Et c'est tout, donc ils ont des outils, ils peuvent candidater, même quand ils sortent sans emploi. Et au niveau des jeunes, jamais je les laisse sans formation, je les oriente toujours sur des formations, avant la fin de l'accompagnement, je mets un point d'honneur, quand je sais qu'ils vont sortir sans emploi, à rechercher avec eux une solution formation, parce qu'en général, s'ils sortent sans emploi, c'est parce qu'ils ont une compétence en moins, ou une chose qui fait que, bon, ça empêche l'insertion. »(OPP X1, conseillère)

En ce qui concerne les méthodes d'activation des chômeurs, mises en œuvre dans le cadre de l'interaction de conseil, des processus semblables à ceux observés entre les chômeurs et les conseillers des organismes classiques ont pu être identifiés, soit à travers leur actualisation à OPP Intérim, soit par défaut à $\mathrm{OPP} \mathrm{X} 1$ et 2 où les conseillères sont aux prises avec des contradictions organisationnelles. Alors qu'elles sont concrètement confrontées aux attentes que suscite une situation d'entretien en face à face (demande d'écoute, récit de soi, confidences personnelles, recherche d'un soutien moral, etc.), elles sont contraintes sans y parvenir, faute de temps, de compétences et d'autorisation formelle, de rationaliser la relation avec le chômeur et de centrer l'échange sur la recherche d'emploi. Même si certains aspects relationnels, comme la responsabilisation ou la culpabilisation du chômeur, peuvent être moralement critiquables, l'évacuation des émotions et l'instauration d'un pragmatisme jugé synonyme d'efficacité montrent, en l'occurrence, que cette définition de la situation d'accompagne- ment ne répond pas aux attentes des chômeurs et complexifie la tâche du conseiller. Cette tendance à la «dépsychologisation» soulève notamment la question du bien fondé de méthodes telles que le profilage des chômeurs qui dépossèdent le conseiller d'une part de ses attributions au nom d'une plus grande objectivité du traitement des chômeurs.

Enfin, il apparaît que le rôle d'intermédiation entre l'offre et la demande d'emploi demeure incomplètement tenu par les conseillers d'OPP Intérim et d'OPP X1 et 2. Chez l'un, les contacts avec les employeurs sont médiatisés et filtrés par les agences de travail temporaires du réseau de la société; chez l'autre, la prospection des offres d'emploi et le démarchage des entreprises se trouvent relégués au second plan, tant les critères des commanditaires donnent la priorité aux taux de retour en emploi et donc au travail pour et sur autrui, c'est-à-dire sur les demandeurs d'emploi.

Les résultats de nos investigations sur les pratiques des nouveaux opérateurs privés demeurent partiels et sommaires; ils demandent à être approfondis, notamment au moment où la direction générale de Pôle Emploi manifeste la volonté d'accentuer l'externalisation de l'accompagnement par le recours à des organismes privés des demandeurs d'emploi. L'augmentation du nombre des chômeurs semble donc accélérer le développement de l'intervention des OPP, offrant ainsi un vaste terrain de recherche où l'expérimentation semble céder le pas à l'institutionnalisation de ces nouveaux prestataires privés de service. 


\section{Bibliographie}

Balmary D. (2004), Rapport de l'instance d'évaluation de la politique de l'emploi et recours à des opérateurs externes, Commissariat général du Plan, Paris, février.

Baron C., Bureau M.-C., Le Dantec E., Nivolle P. (1994), «Les intermédiaires de l'insertion», Paris, CEE, Dossier $n^{\circ} 1$, Nouvelle Série, mai.

Belkacem R., Charlier M., Deshayes J.-L., Hirlet P. (2001), «Intermédiaires de l'emploi ou employeurs territoriaux de main-d'œuvre? », Utinam, $\mathrm{n}^{\circ}$ 5, pp. 40-50.

Benarrosh Y. (2000), «Tri des chômeurs: le nécessaire consensus des acteurs de l'emploi », Travail et Emploi, $\mathrm{n}^{\circ} 81$, janvier, pp. 9-26.

Bureau M.-C., Marchal E. (2005), «Pluralité des marchés du travail et qualités des intermédiaires », Centres d'études de l'emploi, Document de travail, $\mathrm{n}^{\circ} 48$, novembre.

CASTRA D. (2003), L'insertion professionnelle des publics précaires, Paris, PUF.

Chevrier-Fatôme C., Simonin B. (2004) «Politique de l'emploi: un nombre croissant d'intervenants», Premières synthèses, Dares, juillet, 30.2.

Coutant I. (2005), «Le pouvoir des mots: à propos de l'encadrement socio-éducatif des jeunes sans avenir», Formation Emploi, n ${ }^{\circ}$ 89, pp. 19-33.

Crépon B., Dejemeppe M., Gurgand M., (2005) «Un bilan de l'accompagnement des chômeurs», Connaissance de l'emploi, $\mathrm{n}^{\circ} 20$, septembre.

Divay S. (1999), L'aide à la recherche d'emploi. Des conseils pour sauver la face, Paris, L'Harmattan.

Divay S. (2008), «Psychologisation et dépsychologisation de l'accompagnement des chômeurs », Sociologies pratiques, $\mathrm{n}^{\circ} 17$, pp. 55-66.

Ebersold S. (2001), La naissance de l'inemployable. Ou l'insertion au risque de l'exclusion, Rennes, PUR.

Exertier A., Gramain A., Legal A., (2006), «Les modes de coordination des acteurs locaux autour des dispositifs du Pare et du Pap. Une synthèse de l'enquête», Paris, CEE, Document de travail, $\mathrm{n}^{\circ} 64$, juillet.

Ferracci M. (2007), «Améliorer le Service public de l'emploi: ce que disent les faits», Revue française d'économie, $\mathrm{n}^{\circ}$ 3, vol XXI, janvier, pp. 75-135.

Frétigné C. (2004), Une formation à l'emploi?, Paris, L'Harmattan.

Georges N. (2006), «Le profilage: outil statistique et/ou mode de coordination?», Paris, CEE, Document de travail, $\mathrm{n}^{\circ} 72$, novembre.

Georges N. (2007), «L'externalisation de l'accompagnement des demandeurs d'emploi : modalités d'un marché en plein essor », Paris, CEE, Document de travail, $n^{\circ} 81$, février.

Houzel Y., Outin J.-L., Ramaux C. (2000), «Travail, logiques d'action et sens du travail des intermédiaires de l'emploi», in Didier Gélot, Patrick Nivolle (dir.), «Les intermédiaires des politiques publiques de l'emploi», Cahier Travail et Emploi, CEE, Paris, La Documentation française, pp.107-137.

JELlab A. (1997), Le travail d'insertion en mission locale, Paris, L'Harmattan.

MARIMBert J. (2004), Rapport au ministre des Affaires sociales, du Travail et de la Solidarité sur le rapprochement des services de l'emploi, janvier, La Documentation française.

Meyer J.-L. (1998), «Intermédiaires de l'emploi et marché du travail», Sociologie du travail, ${ }^{\circ} 3$, pp. 345364.

Nivolle P. (1999), «Stratégies des acteurs locaux», in Cavestro William, Lamotte Bruno, «Travail et emploi : vers de nouvelles régulations », Cahier Travail et Emploi, CEE, La Documentation française, pp.133-156.

Orianne J.-F. (2004), «Troubles de l'employabilité et traitement clinique du chômage », $4^{\mathrm{e}}$ Conférence intermédiaire du RC52 de l'AIS, Savoirs, travail et organisation, Université de Versailles-Saint-Quentin-en-Yvelines, septembre.

Perez C., Personnaz E. (2008), «Les services d'information, de conseil et d'orientation professionnelle des adultes : un appui aux transitions professionnelles?», Marseille, Céreq, RELIEF, $\mathrm{n}^{\circ} 27$, juillet.

Pochic S. (2007), «Développer la recrutabilité. L'autre versant des transitions professionnelles », Cadres-CFDT, $n^{\circ} 425-426$.

Rousseau Y. (2005), «Du monopole public de placement à un nouveau service public de l'emploi», Droit social, avril.

Salais R., Baverez N., Reynaud B. (1989), L'invention du chômage, Paris, PUF.

Salognon M. (2007), «Le chômage de longue durée: une rupture à combattre autrement pour assurer sa réversibilité», Communication pour les XIVe journées d'études sur les données longitudinales dans l'analyse du marché du travail, Orléans, mai.

Tralongo S. (2008), «La méthode du "Projet personnel et professionnel": un "travail de soi" des étudiants », Sociologies pratiques, $\mathrm{n}^{\circ} 17$, pp. 95-105.

VRancken D., Macquet C. (2006), Le travail sur soi. Vers une psychologisation de la société?, Paris, Éditions Belin. 